Check for updates

Cite this: Chem. Commun., 2020, 56, 13355

Received 25th August 2020,

Accepted 28th September 2020

DOI: $10.1039 / d 0 c c 05745 b$

rsc.li/chemcomm

\section{Chronopotentiometric aptasensing with signal amplification based on enzyme-catalyzed surface polymerization $\dagger$}

\author{
Shuwen Liu, ${ }^{\text {ab }}$ Jiawang Ding ${ }^{\text {abcd }}$ and Wei Qin (D) *abcd
}

\begin{abstract}
A signal amplification strategy based on the horseradish peroxidase catalyzed polymerization of dopamine on a polymeric ion-selective membrane surface is proposed for the sensitive chronopotentiometric detection of an aptamer-target binding event.
\end{abstract}

Signal transductions that rely on ion/charge transfer have been extensively explored for developing sensitive, selective and versatile electrochemical sensors. ${ }^{1-4}$ As one of the most commonly used electrochemical sensors, ${ }^{5-9}$ potentiometric polymeric membrane ion-selective electrodes (ISEs) have received considerable attention through a deep understanding of ion transfer processes across liquid-liquid interfaces in the last two decades. ${ }^{10-12}$ Modulation of ion fluxes across the polymeric membrane can be readily achieved by applying a controlled current or potential. Many attractive methodologies including chronopotentiometry, ${ }^{13-15}$ coulometry, ${ }^{16-18}$ stripping voltammetry $^{19,20}$ and coulometric/chronopotentiometric flash titrimetry $^{21}$ have been developed with a range of improved characteristics. ${ }^{10}$

In recent years, surface confined reactions have been electrochemically monitored by blocking passive ${ }^{22}$ or currentdriven ion fluxes ${ }^{23}$ of polymeric membrane electrodes. For these assays, a surface binding event at the ion-selective membrane/solution interface alters the interfacial ion transport and therefore can be sensed potentiometrically. Various recognition modes such as antibody-antigen interaction, ${ }^{22}$

\footnotetext{
${ }^{a}$ Key Laboratory of Coastal Environmental Processes and Ecological Remediation, Yantai Institute of Coastal Zone Research (YIC), Chinese Academy of Sciences (CAS), Shandong Key Laboratory of Coastal Environmental Processes, YICCAS, Yantai, Shandong 264003, P. R. China.E-mail: jwding@yic.ac.cn,wqin@yic.ac.cn; Fax: +86 535 2109000; Tel: +865352109156

${ }^{b}$ University of the Chinese Academy of Sciences, Beijing 100049, P. R. China

${ }^{c}$ Laboratory for Marine Biology and Biotechnology, Pilot National Laboratory for Marine Science and Technology (Qing-dao), Shandong 266237, P. R. China

${ }^{d}$ Center for Ocean Mega-Science, Chinese Academy of Sciences, Qingdao, Shandong 266071, P. R. China

$\dagger$ Electronic supplementary information (ESI) available: Experimental details and supplementary figures. See DOI: 10.1039/d0cc05745b
}

DNA-DNA hybridization, ${ }^{24}$ and artificial bioreceptor-bioanalyte binding ${ }^{25}$ have been developed based on the surface blocking of ion fluxes across ion-selective membranes (ISMs), which allows the label-free detection of a large number of different molecules other than ionic species. ${ }^{25-27}$ However, effective blocking of the active surface area cannot be easily achieved. ${ }^{28}$ In order to achieve high sensitivity, a dense receptor layer modified on the membrane surface and an effective surface blocking via receptor/analyte binding are always required. However, for these assays, the distribution of the biomolecules immobilized on the membrane surface could be nonuniform and a single target molecule can only bind to a single receptor on the surface. Therefore, for an effective surface coverage, the sensing targets are restricted to biomacromolecules such as antigens/antibodies, proteins and cells, and an indicator ion with a large size is preferred. ${ }^{25}$

Herein, a novel chronopotentiometric aptasensing plaform based on an effective ion transfer blockage induced by an enzyme-catalyzed polymerization is proposed for highly sensitive detection of small molecules with free selection of indicator ions. Dopamine (DA) can polymerize in a weakly alkaline aqueous soultion to form a highly adherent polymer coating on virtually any substrate surface. ${ }^{29,30}$ Owing to the potent adhesion behavior, polydopamine (PDA) has been arguably a promising dip-coating material for surface modification. Moreover, DA polymerization can be accelerated by using horseradish peroxidase (HRP). ${ }^{31}$ Inspired by the unique properties of PDA in surface coating, we demonstrate a chronopotentiometric aptasensing strategy based on HRP-catalyzed surface polymerization of DA that blocks the current-induced ion transfer of an indicator ion from the sample solution to the polymeric ion-slective membrane. This biocatalyzed polymerization can significantly enhance the surface coverage, thus inducing an effective blocking effect.

As a proof of concept experiment, a sensitive and selective chronopotentiometric aptasensing assay based on an enzymecatalyzed polymerization induced blocking mechanism was designed. The sensing principle is illustrated in Scheme 1. 


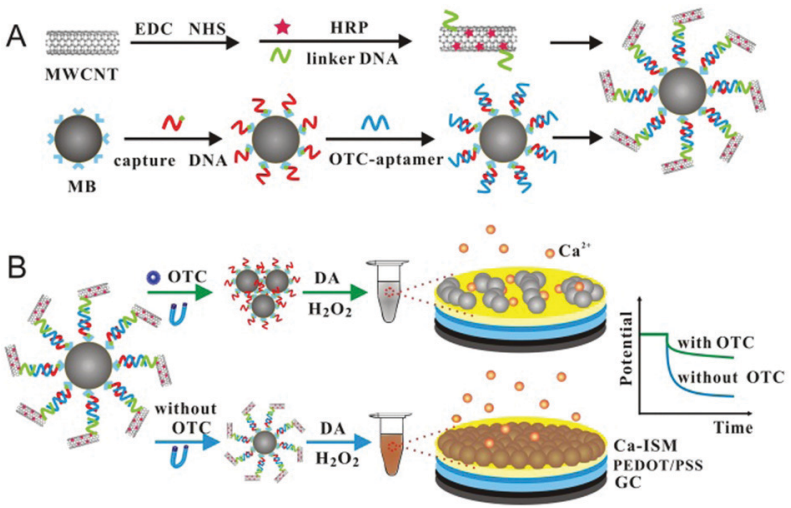

Scheme 1 Schematic illustrations of the synthesis of the MWCNT probe functionalized magnetic beads (A) and the principle of a chronopotentiometric aptasensing platform for OTC detection based on the ion blocking effect (B)

Magnetic beads (MBs) with attractive features in bioseparation were used as the probe carrier. ${ }^{32}$ Oxytetracycline (OTC), an extensively used antibiotic that can result in drug resistance in bacteria, was used as the model target. An aptamer with a sequence of ACGTTGACGCTGGTGCCCGGTTGTGGTGCGAGTGTTGTGT (40 mer) that can bind to OTC with high specificity and good affinity was selected as the recognition element. ${ }^{33}$ As shown in Scheme 1A, the OTC aptamer can hybridize simultaneously with the linker DNA immobilized on the HRP-loaded multi-walled carbon nanotubes (MWCNTs) and with the capture DNA immobilized on the MBs (Fig. S1, ESI + ). The formed sandwich structures can be decomposed by the targetaptamer binding event to release the HRP-loaded MWCNTs.

In the absence of the target, the HRP molecules in the sandwich structures can effectively induce biocatalyzed polymerization of DA. As a result, the formed PDA film strongly adhering to the $\mathrm{Ca}^{2+}$-ISM layer blocks current-induced $\mathrm{Ca}^{2+}$ ion fluxes, and subsequently results in a change in the superNernstian potential response. In the presence of the target, the target-aptamer binding event induces the release of the HRP-labeled MWCNTs from the MBs and thus retards the formation of the PDA film on the membrane electrode surface. The blocking effect induced by the PDA film anchored onto the membrane can be interrogated by pulsed galvanostatic ion extraction of $\mathrm{Ca}^{2+}$ into the polymeric membrane. By measuring the chronopotential changes of the $\mathrm{Ca}^{2+}$-ISM coated with PDA in the absence and presence of the target, OTC can be detected (Scheme 1B). For the proposed assay, multiple HRP molecules are loaded onto the carbon nanotubes and the enzymecatalyzed polymerization of DA is involved, both of which could allow rapid localized deposition of the PDA film on the surface of the polymeric membrane, thus dramatically enhancing the detection sensitivity.

The carbon nanotube segments with multiple immobilized HRP labels were used for signal amplification. For preparation of the MWCNT-HRP-linker DNA complexes, the linker DNA and HRP were tethered onto the MWCNT surface via covalent binding of the $-\mathrm{NH}_{2}$ groups of the linker DNA and HRP with the
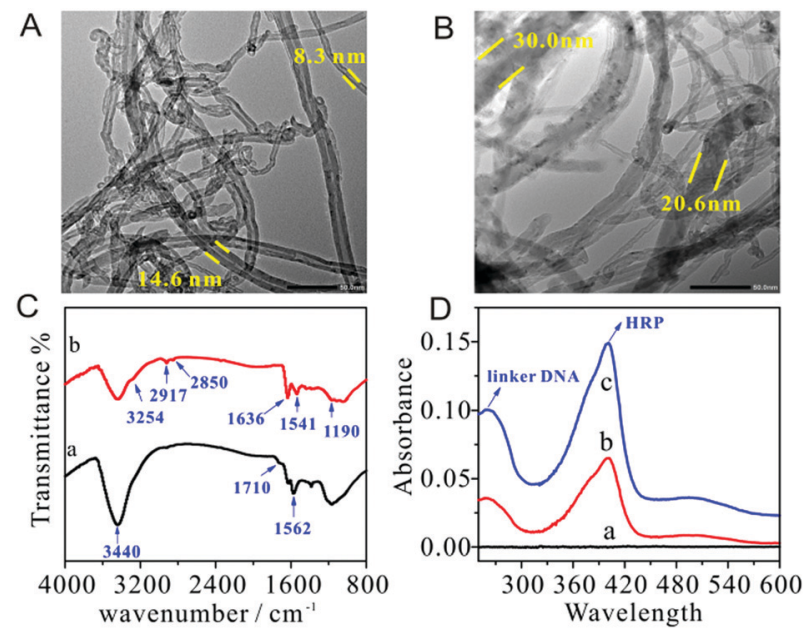

Fig. 1 TEM images ( $A$ and $B$ ) and Fourier transform infrared (FTIR) spectra (C) of the MWCNTs ( $A$; $a$ in $C$ ) and the MWCNTs conjugated with the linker DNA and HRP (B; $b$ in $C$ ). (D) UV-vis spectra of the blank (buffer) (a), the mixture solution with $1 \mu \mathrm{M}$ of linker DNA and $12.5 \mu \mathrm{M}\left(0.5 \mathrm{mg} \mathrm{mL}^{-1}\right)$ of HRP after (b) and before (c) incubation with $300 \mu \mathrm{L}$ of MWCNTs for $6 \mathrm{~h}$. UV-vis spectroscopic measurements were done in 0.01 M PBS buffer of pH 7.4.

- $\mathrm{COOH}$ groups of the MWCNTs. The transmission electron microscopy (TEM) image reveals that the diameters of pristine MWCNTs are about 8-15 nm (Fig. 1A), whereas a diameter of about 20-30 nm is observed for the MWCNTs loaded with DNA and HRP (Fig. 1B). The enzyme aggregates appearing on the side walls of the nanotubes may lead to the increased diameters. ${ }^{34}$ As indicated in Fig. 1C, the FTIR spectra also reveal that HRP (typical characteristics at 2917, 2850, 1636 and $1541 \mathrm{~cm}^{-1}$ ) and the linker DNA (small $\mathrm{PO}_{2}$ stretching band at $1190 \mathrm{~cm}^{-1}$ ) were covalently attached on the surface of the MWCNTs (the corresponding FTIR spectra of HRP and the linker DNA are shown in Fig. S2, ESI $\dagger$ ). ${ }^{35}$ In addition, the disappearance of the stretching vibration of $\mathrm{C}=\mathrm{O}$ at $1710 \mathrm{~cm}^{-1}$ implies the efficient reaction of $-\mathrm{COOH}$ in MWCNTs and the successful linking of the linker DNA and HRP to MWCNTs. As proved from the UV-vis spectra, the linker DNA (at $260 \mathrm{~nm}$ ) and HRP (at $400 \mathrm{~nm}$ ) are immobilized on the MWCNTs (Fig. 1D).

Pulsed galvanostatic chronopotentiometry, which can rapidly and accurately control ion extraction processes, ${ }^{36}$ was used as an effective tool to monitor the change in the calcium ion fluxes from the sample to the sensing membrane. Compared with the conventional potential responses of $\mathrm{Ca}^{2+}$ (Fig. S3, ESI $\dagger$ ), the ion transfer blocking effect is much more pronounced in the so-called super-Nernstian concentration range around $10^{-4} \mathrm{M}$, which is indicative of $\mathrm{Ca}^{2+}$ depletion in the vicinity of the membrane surface induced by the cathodic current pulse. ${ }^{23}$ Therefore, in order to monitor the calcium ion blocking effect with high sensitivity, the potential difference of $10^{-4} \mathrm{M} \mathrm{Ca}^{2+}$ concentrations in the super-Nernstian range was tested by pulsed galvanostatic chronopotentiometry for a quantitative measurement. Moreover, the blocking effect is significantly enhanced by the high coverage of the membrane surface via the HRP-caltalyzed DA polymerization (Fig. 2). Indeed, both UV-vis absorbances (Fig. S4, ESI $\dagger$ ) and the charge 


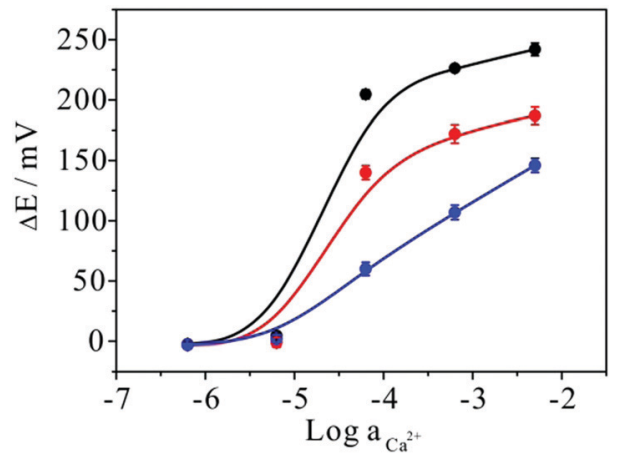

Fig. 2 Pulsed chronopotentiometric responses of the $\mathrm{Ca}^{2+}-$ ISEs to $\mathrm{Ca}^{2+}$ in $0.01 \mathrm{M} \mathrm{NaCl}$ without the dopamine polymerization (in black), with the dopamine autopolymerization (in red), and with the HRP-induced dopamine polymerization (in blue) for $45 \mathrm{~min}$. For the HRP-induced dopamine polymerization, $100 \mu \mathrm{L}$ of the blocking buffer solution containing $7.5 \mathrm{mg} \mathrm{mL}^{-1}$ dopamine, $3.2 \times 10^{-3} \mathrm{U} \mathrm{mL}^{-1} \mathrm{HRP}$, and $20 \mathrm{mM} \mathrm{H}_{2} \mathrm{O}_{2}$ was drop-coated on the $\mathrm{Ca}^{2+}-$ ISM. Error bars represent one standard deviation for three measurements.

transfer resistance (Fig. S5 ESI $\dagger$ ) reveal that the presence of HRP and $\mathrm{H}_{2} \mathrm{O}_{2}$ can rapidly trigger the formation of PDA. The formed PDA film on the electrode demonstrates an obvious potential difference in between the absence and presence of HRP. The change in the potential as a function of concentration of HRP was found to be linear in the range of $5.0 \times 10^{-4}-2.5 \times 10^{-2} \mathrm{U} \mathrm{mL}^{-1}$ with a detection limit of $3.0 \times 10^{-4} \mathrm{U} \mathrm{mL}^{-1}(3 \sigma / S$, Fig. S6, ESI $\dagger)$.

It should be noted that although different substrates such as epinephrine, aniline and dopamine can be polymerized and deposited on the membrane in the presence of HRP, PDA can strongly adhere to the electrode and show the best sensitivity (Fig. S7, ESI $\dagger$ ). The experimental parameters such as the DA concentration, DA polymerization time, and $\mathrm{pH}$ of the buffer solution and the pulsed current parameters were optimized. In order to reduce the autopolymerization of DA and achieve a large change in the potential, $7.5 \mathrm{mg} \mathrm{mL}^{-1} \mathrm{DA}$ with a polymerization time of $45 \mathrm{~min}$ at $\mathrm{pH} 8.0$ and a cathodic current amplitude of $2 \mu \mathrm{A}$ with a duration of $2 \mathrm{~s}$ were selected for further experiments (Fig. S8 and S9, ESI $\dagger$ ).

In this work, the enzymatically biocatalyzed polymerization of DA is essential for blocking the $\mathrm{Ca}^{2+}$ fluxes on the electrode surface. As proved from the chronopotentiometric measurements (Fig. 3A), the MBs, MWCNTs/HRP/linker DNA and MBsOTC-Apt/linker DNA/MWCNTs/HRP without the addition of DA could not block the $\mathrm{Ca}^{2+}$ flux (red). In contrast, in the presence of DA, the blocking of the $\mathrm{Ca}^{2+}$ flux occurs (blue) and a significant potential difference decrease was observed with MWCNTs/HRP ( $c$ and $d$ in blue). The potential difference decrease could be mainly ascribed to the effective blocking of the calcium ion fluxes across the membrane by PDA, which is catalyzed by HRP on the surface of the PVC membrane. The contact angle (CA) measurements also demonstrate such a difference. As shown in Fig. 3B, because of the hydrophilic nature of PDA, the PDA treatment could lead to a visible change in the PVC surface wettability.

Under the optimized conditions, the analytical performance of the proposed sensing strategy was demonstrated for the
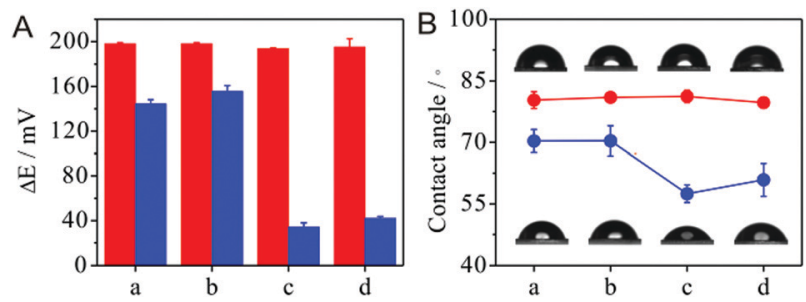

Fig. 3 (A) Pulsed chronopotentiometric responses to $10^{-4} \mathrm{M} \mathrm{Ca}^{2+}$ in $0.01 \mathrm{M} \mathrm{NaCl}$ in the absence (in red) or presence of PDA blockage (in blue). (B) Contact angles of the calcium-selective membrane surfaces before (in red) and after (in blue) PDA blockage. The calcium-selective membranes were coated with $100 \mu \mathrm{L}$ of PBS (a), $1.0 \mathrm{mg} \mathrm{mL}^{-1} \mathrm{MBs}$ (b), MWCNTs/HRP/ linker DNA (c) and MBs-OTC-Apt/linker DNA/MWCNTs/HRP (d) for characterization. The polymerization conditions were as follows: $0.01 \mathrm{M} \mathrm{pH}$ 8.0 PBS, $7.5 \mathrm{mg} \mathrm{mL}^{-1}$ dopamine, $20 \mathrm{mM} \mathrm{H}_{2} \mathrm{O}_{2}$ and 45 min polymerization time. Error bars represent one standard deviation for three measurements.

detection of OTC. The presence of target OTC triggers the release of the HRP-labelled MWCNTs, which dramatically decelerates the PDA precipitation on the $\mathrm{Ca}^{2+}$-ISM surface, thus leading to a potentiometric signal increase. As shown in Fig. 4A, the potential difference of the $\mathrm{Ca}^{2+}$-ISE for measuring $10^{-4} \mathrm{M}$ $\mathrm{Ca}^{2+}$ significantly increased with the increasing concentration of OTC. A linear relationship $\left(\Delta E=19.90 \times \log C_{\text {OTC }}+218.37\right.$, $R^{2}=0.99$ ) between the OTC concentration and the potential difference was observed in a wide concentration range from 0.1 to $1000 \mathrm{nM}$, exhibiting a detection limit down to $28 \mathrm{pM}(3 \sigma / S$, Fig. 4B). This excellent sensitivity is better than those of other
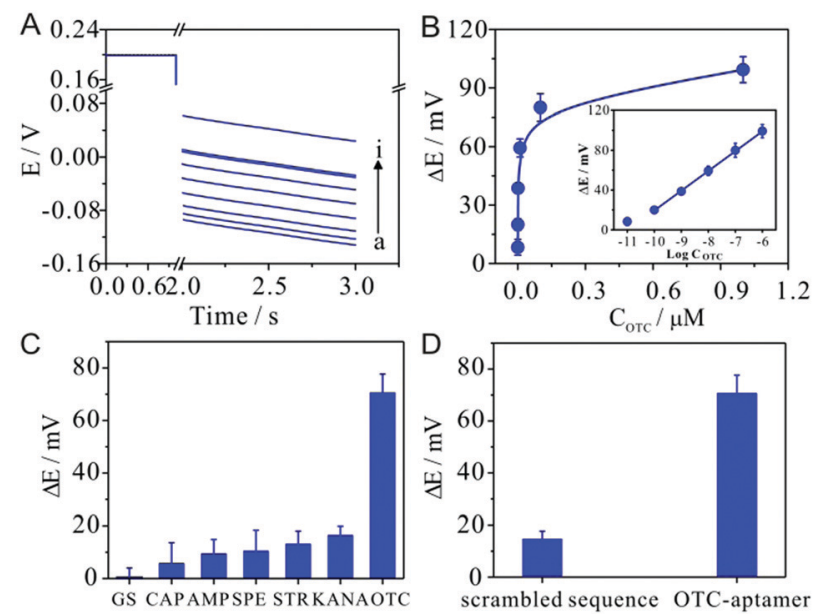

Fig. 4 (A) Pulsed chronopotentiometric responses of the calciumselective electrode measuring $10^{-4}(a-i) \mathrm{M} \mathrm{Ca}^{2+}$ in the presence of 0 (a), $10^{-11}$ (b), $10^{-10}$ (c), $10^{-9}$ (d), $10^{-8}$ (e), $10^{-7}$ (f) and $10^{-6}$ (g) M of OTC. The membranes blocked by dopamine autopolymerization (h) and not blocked (i) were investigated. (B) Corresponding calibration plot for OTC detection. The chronopotential difference $(\Delta E)$ between the responses with $(b-g)$ and without OTC (a) was used for quantification. (C) Responses to the targets and other interfering antibiotics including CAP, STR, AMP, GS, SPE and KANA. The concentration of OTC was $100 \mathrm{nM}$, and those of the other antibiotics were $1 \mu \mathrm{M}$. (D) Responses to OTC by using the OTC-aptamer and a scrambled DNA sequence. All experiments were done in $\mathrm{PBS}$ at $\mathrm{pH}$ 8.0 with $7.5 \mathrm{mg} \mathrm{mL}^{-1}$ dopamine, $20 \mathrm{mM} \mathrm{H}_{2} \mathrm{O}_{2}$ and 45 min polymerization time. Error bars represent one standard deviation for three measurements. 
reported OTC sensors (Table S2, ESI $\dagger$ ). The proposed assay shows good selectivity of OTC over other antibiotics including chloramphenicol (CAP), streptomycin (STR), ampicillin (AMP), gentamycin (GS), spectinomycin (SPE) and kanamycin (KANA) (Fig. 4C). In addition, an oligonucleotide strand with a scrambled DNA sequence was selected as the control probe to validate the specificity of this method. It was found that no significant change in the potential was observed for the scrambled DNA sequence (Fig. 4D).

To validate the practicability, sea water samples collected from the Bohai Sea $\left(121.0-121.3^{\circ} \mathrm{E}, 37.9-38.6^{\circ} \mathrm{N}\right)$ were analyzed by using the proposed potentiometric aptasensing platform with the standard addition method. The obtained recoveries ranged from 94 to $101 \%$ and the standard deviations were in the range of 5 to $8 \%$, which indicates that the proposed potentiometric aptasensing platform is promising for practical analysis (Table S3, ESI $\dagger$ ). Moreover, the electrode shows reproducible potential differences at $10^{-4} \mathrm{M} \mathrm{Ca}^{2+}$ and can be used at least for 3 cycles (Fig. S10, $\mathrm{ESI}^{\dagger}$ ).

In summary, a chronopotentiometric aptasensor based on signal amplification for highly sensitive and selective detection of oxytetracycline using $\mathrm{Ca}^{2+}$ as the indicator ion is proposed. Unlike the reported sensors based on the blocking effect induced by a surface binding event, the in situ enzyme-catalyzed polymerization induced blocking for potentiometric sensing enables the detection of small molecules with high sensitivity and free selection of indicator ions. Compared to other OTC sensors, the present sensing strategy exhibits a detection limit down to 28 pM. This enhanced sensitivity is due to the combination of the following mechanisms: (1) the signal is significantly amplified by using MWCNTs with a high surface area and functional carboxylic groups to load a large amount of HRP, which catalyzes the PDA polymerization and (2) the super-Nernstian response range for the $\mathrm{Ca}^{2+}$ measurements using chronopotentiometry is highly sensitive to the surface blocking events. Considering the effective blockage of the extracted ion fluxes, the chronopotentiometric aptasensing platform can be extended to detect other analytes for biosensing and environmental analysis simply by changing the corresponding aptamers.

This work was financially supported by the National Natural Science Foundation of China (41876108 and 21677172), the Instrument Developing Project of the Chinese Academy of Sciences (Y728021021), the National Key Research and Development Program of China (No. 2016YFC1400700), the Taishan Scholar Program of Shandong Province (tspd20181215 and tsqn201909163) and the Key Research and Development Program of Yantai (2018ZHGY053).

\section{Conflicts of interest}

There are no conflicts to declare.

\section{Notes and references}

1 K. J. Cash, F. Ricci and K. W. Plaxco, J. Am. Chem. Soc., 2009, 131, 6955-6956.

2 Y. L. Ying, Y. X. Hu, R. Gao, R. J. Yu, Z. Gu, L. P. Lee and Y. T. Long, J. Am. Chem. Soc., 2018, 140, 5385-5392.

3 M. Cuartero, R. G. Acres, R. De Marco, E. Bakker and G. A. Crespo, Anal. Chem., 2016, 88, 6939-6946.

4 M. Podrazka, E. W. Nery, A. Pacowska, D. W. M. Arrigan and M. Jonsson-Niedziolka, Anal. Chem., 2018, 90, 8727-8731.

5 E. Bakker and E. Pretsch, Angew. Chem., Int. Ed., 2007, 46, 5660-5668.

6 J. Bobacka, A. Ivaska and A. Lewenstam, Chem. Rev., 2008, 108, 329-351.

7 J. B. Hu, A. Stein and P. Bühlmann, TrAC, Trends Anal. Chem., 2016, 76, 102-114.

8 E. Zdrachek and E. Bakker, Anal. Chem., 2019, 91, 2-26.

9 J. W. Ding and W. Qin, TrAC, Trends Anal. Chem., 2020, 124, 115803.

10 E. Bakker, TrAC, Trends Anal. Chem., 2014, 53, 98-105.

11 E. Bakker, Anal. Chem., 2016, 88, 395-413.

12 X. U. Zou and P. Bühlmann, Anal. Chem., 2013, 85, 3817-3821.

13 É. Pergel, R. E. Gyurcsányi, K. Tóth and E. Lindner, Anal. Chem., 2001, 73, 4249-4253.

14 A. Shvarev and E. Bakker, Anal. Chem., 2003, 75, 4541-4550.

15 J. W. Ding and W. Qin, J. Am. Chem. Soc., 2009, 131, 14640-14641.

16 E. Grygolowicz-Pawlak and E. Bakker, Anal. Chem., 2010, 82, 4537-4542.

17 U. Vanamo, E. Hupa, V. Yrjana and J. Bobacka, Anal. Chem., 2016, 88, 4369-4374.

18 Z. Jarolimova, T. Han, U. Mattinen, J. Bobacka and E. Bakker, Anal. Chem., 2018, 90, 8700-8707.

19 H. Katano and M. Senda, J. Electroanal. Chem., 2001, 496, 103-109.

20 Y. Kim, P. J. Rodgers, R. Ishimatsu and S. Amemiya, Anal. Chem., 2009, 81, 7262-7270.

21 V. Bhakthavatsalam, A. Shvarev and E. Bakker, Analyst, 2006, 131, 895-900.

22 M. S. Ozdemir, M. Marczak, H. Bohets, K. Bonroy, D. Roymans, L. Stuyver, K. Vanhoutte, M. Pawlak and E. Bakker, Anal. Chem., 2013, 85, 4770-4776.

23 Y. D. Xu and E. Bakker, Langmuir, 2009, 25, 568-573.

24 T. V. Shishkanova, R. Volf, M. Krondak and V. Kral, Biosens. Bioelectron., 2007, 22, 2712-2717.

25 R. N. Liang, J. W. Ding, S. S. Gao and W. Qin, Angew. Chem., Int. Ed., 2017, 56, 6833-6837.

26 M. Pawlak, G. Mistlberger and E. Bakker, Microchim. Acta, 2014, 182, 129-137.

27 A. H. N. Medhat Ahmed Al-Ghobashy, G. M. El-Sayed and M. Nebsen, ACS Sens., 2019, 4, 413-420.

28 P. Reichmuth, H. Sigrist, M. Badertscher, W. E. Morf, N. F. de Rooij and E. Pretsch, Bioconjugate Chem., 2002, 13, 90-96.

29 H. Lee, S. M. Dellatore, W. M. Miller and P. B. Messersmith, Science, 2007, 318, 426-430.

30 Y. L. Liu, K. Ai and L. H. Lu, Chem. Rev., 2014, 114, 5057-5115.

31 J. W. Li, M. A. Baird, M. A. Davis, W. Y. Tai, L. S. Zweifel, K. M. A. Waldorf, M. Gale, L. Rajagopal, R. H. Pierce and X. Gao, Nat. Biomed. Eng., 2017, 1, 0082.

32 L. H. Reddy, J. L. Arias, J. Nicolas and P. Couvreur, Chem. Rev., 2012, 112, 5818-5878.

33 J. H. Niazi, S. J. Lee and M. B. Gu, Bioorg. Med. Chem., 2008, 16, $7245-7253$.

34 Q. Z. Zhang, B. Zhao, J. Yan, S. P. Song, R. Min and C. H. Fan, Anal. Chem., 2011, 83, 9191-9196.

35 J. Li, J. J. Wang, X. Guo, Q. Zheng, J. Peng, H. Tang and S. Z. Yao, Anal. Chem., 2015, 87, 7610-7617.

36 Y. Xu, R. De Marco, A. Shvarev and E. Bakker, Chem. Commun., 2005, 3074-3076. 